\title{
Spectrum and Prevalence of degenerative changes seen on Mri of Lumbosacral Spine in Patients with Low Back Pain
}

\author{
Dr. Sai Sudha Angam ${ }^{1}$,Dr. Nadeem Ahmed ${ }^{2}$,Dr. Sreedevi Thakkallapelli ${ }^{3}$, \\ Dr.Swapna Chouhan ${ }^{4}$,Dr. Madhavilatha Routhu ${ }^{5}$,Dr. Jagdeesh Babu ${ }^{6}$ \\ ${ }^{1}$ Post- graduate Resident ${ }^{2}$ Associate Professor ${ }^{3,4}$ Assistant Professor ${ }^{5,6}$ Tutor, Department of Radiodiagnosis, \\ Kakatiya Medical College, MGM Hospital, Warangal, Telangana.
}

\begin{abstract}
Low back pain (LBP) is the most prevalent musculoskeletal condition and one of the most common causes of disability. In a report of World Health Organization in 2002, LBP constituted 37\% of all occupational risk factors and occupies first rank among the disease complications caused by work. Magnetic resonance imaging (MRI) with its multiplanar capabilities and lack of ionizing radiation provides excellent anatomical details of the lumbar spine and detects the cause of LBP in a majority of patients. This cross sectional study was carried out in Radiology Department of MGMH, from 2015-2016. The study included 192 patients who presented with low back pain. All patients underwent lumbar MRI using 1.5 T scanner. The current study evaluates different degenerative changes on MRI in patients with LBP. A review of 192 MRIs of patients presenting with LBP established disc bulge as the most common finding (in 85\%), disc dessication (in 66\%), disc protrusion (in 23\%), disc extrusion (in 2\%), HIZ/annular tear (in 22\%) and Schmorl's nodes (in 10\%). Other vertebral degenerative changes include osteophytes (in 52\%), facetal arthropathy (in 27\%) and Modic end plate changes (in 21\%). Flaval hypertrophy is seen in 9\%. Spinal stenosis, which is multifactorial, was seen in 50.5\%. Other less frequently encountered but still significant findings include: vertebral compression (in 5.7\%), transitional vertebra (in 4\%) and spinal listhesis (in 3\%).
\end{abstract}

Keywords: degenerative disease, disc herniation, low back pain, lumbosacral spine, magnetic resonance imaging.

\section{Introduction}

Low back pain (LBP) is the most prevalent musculoskeletal condition and one of the most common causes of disability [1].In accordance with the report of WHO in 2002, LBP constituted 37\% of all occupational risk factors and is among the major disease complications caused by work. Such high prevalence of complications at international levels has made the WHO to name the first decade of the third millennium as the decade of campaign against musculoskeletal disorders -"the silent epidemic" [1].Patients presenting with LBP require diagnostic imaging to determine the cause. Frequently ordered for LBP are plain spine radiographic examinations .These are cost effective and readily available. The limitations are low yield of findings that alter management, poor relationship between most radiographic abnormalities and symptoms of LBP and high doses of gonadal irradiation [2] .This necessitates evaluation by MRI which detects the cause of LBP in a majority of patients. The present study was undertaken to determine the MRI patterns and the common sites of spinal degenerative lesions among patients with low back pain.

\section{Materials And Methods}

The present study was conducted on patients with low backache referred to department of Radiology, MGM Hospital, Warangal for MRI of lumbosacral spine. This was a hospital based cross sectional study conducted from December 2015 to November 2016. Institutional ethics committee permission was obtained to conduct the study. Patients name, age, sex and detailed history were obtained. MRI of the lumbar spine were performed with a $1.5 \mathrm{~T}$ (GE) MR imager using spine phased array coils. The scans consisted of axial T2weighted, sagittal T1and T2-weighted and coronal STIR images with slice thickness of $4.5 \mathrm{~mm}$ for coronal, $4 \mathrm{~mm}$ for axial and $3.5 \mathrm{~mm}$ for sagittal images . A field of view of $32 \times 32 \mathrm{~mm}$ for sagittal and coronal images and $20 \times 20 \mathrm{~mm}$ for axial images were used. The images were stored directly as DICOM files in the workstation.

2.1 Inclusion Criteria- All patients with LBP referred for MRI lumbosacral spine.

2.2 Exclusion Criteria- Patients with acute trauma, infections, neoplastic disease, prior lumbar spine surgery, metallic implants and pacemakers were excluded.

2.3 Statistical Analysis -Statistical analysis was done using SPSS 15 software. Percentages were calculated for the various categories. Chi-square was used to compare the various groups and a $p$-value of $<0.05$ was considered as statistically significant. 


\section{Results}

There were 192 patients of LBP referred for MRI who were included in this study. Out of the total patients there were 90 males $(45.9 \%)$ and $106(54.1 \%)$ females. The age of the patients ranged from 21 years to 80 years. Maximum number of patients were found in the age group between 31 to 40 years.(31.6\%).TABLE 1 shows the frequency of various abnormalities seen on MRI in degenerative disc disease. Disc bulge was the most common finding comprising $85 \%$ of the cases (Fig-1) followed by disc dessication (in 66\%), disc protrusion (in 23\%), and disc extrusion (in 2\%) (Fig-2) HIZ/annular tear was seen in 22\% (Fig-4) and Schmorl's nodes in $10 \%$ of the patients. Other vertebral and degenerative changes included osteophytes (in 52\%), facetal arthropathy (in 27\%), Modic end plate changes (in 21\%)(Fig-4) and Flaval hypertrophy( in 9\%) (Fig-3). Spinal stenosis, which is multifactorial, was seen in $50.5 \%$ of patients. Other less frequently encountered but still significant findings included: vertebral compression (in 5.7\%), transitional vertebra (in 4\%) and spinal listhesis (in 3\%) of the cases.

In the present study of MRI findings in patients with low back pain, disc bulge was the most common abnormality (85\%) and was studied in detail (TABLE-2). Most common level of disc bulge was L4-L5 (in $36.7 \%$ ) followed by L5-S1 (in 26\%) of the cases. Amongst patients with disc bulge, single level involvement was seen in 36 patients (22\%) and multiple level involvement in 127 patients (78\%). Disc herniation was seen in $25 \%$ of the cases (TABLE-3). Among herniated discs, majority (90\%) were disc protrusion and only $10 \%$ were disc extrusion. No cases of disc sequestration were found. Disc protrusion was present in $23 \%$ of total study population, most commonly involving L4-L5 (45\%) and L5-S1 (32\%) levels. In present study, posterocentral disc protrusion $(63.6 \%)$ was most common. There was equal preponderance of disc prolapse in males and females. Most common age of presentation for protruded disc was 31-40yrs (30\%). Similarly the most common age of presentation for extruded disc was 31-40 years (50\%). Marginal osteophytes were noted in 100 patients (52\%) comprising 70 females and 30 males.

Spinal stenosis (including nerve root compression) was present in 50.5\% of cases. Males (51\%) and females (49\%) were almost equally affected. L4-L5 (34\%) intervertebral discs were most frequently affected by spinal stenosis followed by L3-L4 (in 31\%) and L5- S1 (in 21\%). Spinal stenosis can be single or multilevel, and unilateral or bilateral. Multilevel involvement and bilaterality was the most common pattern in this study. Spinal stenosis can be classified as central, lateral recess or foraminal. (TABLE-4) Present study revealed bilateral lateral recess stenosis in 63\% followed by bilateral foraminal stenosis in $20 \%$ and central stenosis in $11 \%$. Depending on the extent of the pathology, central, lateral and foraminal stenosis can occur alone or in combination. Facet joint hypertrophy was observed in $27 \%$ of the study population.Patients of 51-70 year age group accounted for $44 \%$ of involved cases.

Modic end plate changes were seen in $21 \%$ of the present study population. Type I changes were seen in $1.5 \%$ and type II changes in $19.5 \%$; no type III changes were observed. Focal hyperintensity on T2W MRI in posterior annulus of intervertebral disc described as High Intensity Zone (HIZ) was seen in $22 \%$ cases of the study population. Schmorl's nodes were noted in $10 \%$ of the patients. Most common presenting age was 21 $40 \mathrm{yr}$ with majority in males $(80 \%)$ ). Vertebral compression is seen in $5.7 \%$ of the study population with anterior wedge collapse accounting for $90 \%$ of cases.

Lumbosacral transitional vertebrae in the form of sacralization of L5 vertebra was noted in 4\%. No cases of lumbarization of S1 were seen. Spondylolisthesis was noted in 3\% of cases, with female predominance ( $2.5 \%$ female, $0.5 \%$ male). Half of the listhesis cases involved L4-L5 level and half L5-S1 level. When gender is considered female predominance was noted in (83\%) cases. Amongst females, most frequent level of listhesis was L5 over S1 (60\%).

Statistical analysis showed that most common level of disc bulge was in the region of L4-L5 (in 36.7\% cases) as compared to other areas and the difference was statistically significant with $\mathrm{p}$ value $<0.001$. In present study, posterocentral disc protrusion (63.6\%) was most common as compared to other types and the difference was statistically significant with a $p$ value $<0.001$. Disc dessication was more common in females $(41 \%)$ as compared to males $(23 \%)$ and the difference was statistically significant with a $p$ value $<0.001$.

\section{Discussion}

Low back pain (LBP) is the most prevalent musculoskeletal condition and one of the most common causes of disability. The sensitivity of MRI for diagnosing complications resulting from degenerative disc disease like stenosis and nerve root compression is high (81\%-97\%) [3]. The main objective of the current study was to identify the different MRI patterns of disease in LBP patients, determining the most common MRI patterns and the site of lesions. The gender distribution seen in this study showed an overall female preponderance ( $45.9 \%$ males and $54.1 \%$ females). This is in conformity with studies done by Mohd Nazeer et al[4]on low back pain in South Indians where females were 54\% and males $46 \%$. Low back pain has also been associated with hormonal changes, irregular or prolonged menstrual cycles, different pain perception and recall of symptoms [5]. Hence LBP is probably more common in females. Present study finds the highest number of 
patients with LBP to be in their 3rd decade (31.6\%), an age group that corresponds to a large component of the working population. Similar result was found by R Prasad et al who found the most common age of presentation was $31-40$ yrs $(33.3 \%)$ [6].

In the present study of MRI findings in patients with low back pain, disc bulge was the most common abnormality $(85 \%)$. In a study by Younis et al, disc bulge was most frequent finding $(74 \%)$ in patients with low back ache[7].Most common level of disc bulge was L4-L5 (in 36.7\%) followed by L5-S1 (in 26\%). In retrospective study on patterns of requests and findings in MRI of the lumbosacral spine done by Mustapha $\mathrm{Z}$ et $\mathrm{Al}[8]$ disc bulge at L4/L5 IVD level was the most common (34.87\%) followed by L5/S1 (23.28\%). This can be due to increased stress at these lower lumbar levels of the spine compared to higher levels. In patients with disc bulge, single level involvement was seen in 36 patients (22\%) and multiple level involvement was noted in 127 patients (78\%). The study done by Bhutta et al[9]showed single disc involvement in 35\% and multiple disc involvement in $65 \%$ of patients. However, Younis et al[7] found equal incidence of single disc involvement and multiple disc involvement in patients with degenerative spine.

Disc dessication was noted in $66 \%$ of patients. It results from replacement of the glycosaminoglycans within the nucleus pulposus with fibrocartilage which causes reduced disc height and decreased intensity on T2W images[10]. In a similar study done by Osama Al-saeed[11] on MRI of the lumbar spine in young Arabs with low back pain most common feature observed was disc bulge $(88 \%)$ followed by reduced signal intensity of the disc $(82 \%)$. In contrast, disc dessication was the most frequent finding seen in $83 \%$ patients in study of Mboka Jacob et al[12] . This difference could be due to more number of young adults in the present study.

Disc displacement is also a common finding in lumbar spinal degenerative disease. The displaced disc can be just a simple bulge or a herniation. Herniated discs can undergo protrusion, extrusion or sequestration. In this study disc bulge $(85 \%)$ was more common than herniation (25\%). This is different to the findings of Jacob et al [12] who reported more disc herniations than disc bulges in the entire study population. This difference could be due to predominance of younger age group (21-40) in present study. This is supported by the same study of Jacob et al [12] where disc bulges were more common among young individuals aged 20 to 39 years $(40 \%)$ as compared to individuals aged 60 to 80 years $(32 \%)$ and disc herniation was higher among older individuals.

Disc herniation was seen in $25 \%$ in this study, which is similar to study of Uduma et al [13] which showed herniations in $33.3 \%$ of their patients. Among herniated discs, majority (90\%) of types of herniation was disc protrusion; only $10 \%$ were disc extrusion, similar to study of Jacob et al [12]. In present study, disc protrusion was present in $23 \%$ of total study population, most commonly involving L4-L5 (45\%) and L5-S1 $(32 \%)$ levels. The study conducted by Rai et al[14] on MRI findings in chronic low back pain revealed disc protrusion in 23\%, most commonly involving L5/S1 (35\%) level followed by L4/L5 level (30\%). In a study on lumbar disc prolapse by Prasad et al [6], L4-L5 level disc prolapse was most common (34.4\%) followed by L5$\mathrm{S} 1(26.7 \%)$. Various studies have reported that disc herniation is common at L4/L5 and L5/S1 and the frequency at these levels was ranging from $30 \%$ to over $90 \%$.This is also reflected in this study as $76 \%$ of the herniated discs were at L4/L5 and L5/S1 levels.

In present study, posterocentral disc protrusion $(63.6 \%)$ was most common $(p<0.001$, significant), in keeping with findings of Rai et al [14] where $83 \%$ had posterocentral disc protrusion. Study of spine degenerative disease by Jacob et al [12] showed posterolateral disc herniation as the (49\%) most common location.

Marginal osteophytes were noted in 100 patients (52\%), 70 female 30 male. This finding is similar to the study done by Rai et al [14], where $48 \%$ patients had osteophytosis. Osteophytes may form in the absence of other degenerative processes, and cartilaginous damage may exist without corresponding osteophytes. Although there remains a strong association between the presence of osteophytes and other degenerative spine changes, isolated instances of one without the other occur, in the absence of overt symptoms [15].

Lumbar spinal stenosis (including nerve root compression) was present in $50.5 \%$ of cases, this finding was correlating with study of Yong et al where $42.1 \%$ cases had spinal canal stenosis [16], whereas study by M. Jacob [12] revealed lower number (30\%). Both males (51\%) and females (49\%) were almost equally affected. In this study L4-L5 (in 34\%) spinal discs were most frequently affected by LSS, followed by L3-L4 (in 31\%), L5-S1 (in 21\%); this is similar to findings of study done by Uduma et al [13]. LSS can be single or multilevel, and unilateral or bilateral. Multilevel involvement and bilaterality was most common finding in this study. Spinal stenosis can be classified as central, lateral recess or foraminal. Present study revealed bilateral lateral recess stenosis in $63 \%$ followed by bilateral foraminal stenosis in $20 \%$ and central stenosis in $11 \%$. Depending on the extent of the pathology, central, lateral and foraminal stenosis can occur alone or in combination.

Facet joint hypertrophy was observed in $27 \%$, correlating with the study done by PY Yong et al [16] in which $29.8 \%$ had facet arthropathy. Patients of 51-70 year age group were more commonly affected; this finding is supported by studies done by Saleem et al [17] and Rai et al [14]. Modic end plate changes were seen in $21 \%$ of the present study population. Type I changes were seen in $1.5 \%$ and type II changes in $19.5 \%$ of the 
population; no type III changes were observed. $27 \%$ of patients in the study done by Rai et al [14]showed Modic end plate changes, mostly Type I and type II.

Focal hyperintensity on T2W MRI in posterior annulus of the IVD is described as High Intensity Zone (HIZ). HIZ is highly indicative of disc annular tear; some authors [18-20] consider it as a reliable marker for a painful disc while others [21-23] do not. HIZ was seen in $22 \%$ cases of the study population correlating with studies done by Yong et al [16] who found it in 24.6\%. Schmorl's nodes are herniated discs in the craniocaudal (vertical) direction through a break in the vertebral body endplate and are referred to as intravertebral herniations. They were noted in $10 \%$ of the patients in the present study correlating with William et al [24] who found SN in $12 \%$ of study population. Though SN is a common finding on spinal MRI, their prevalence is highly variable depending on the ethnicity and the occupation of the population under study. Most common presenting age in this study was 21-40yr. Schmorl's nodes seem to be commoner in younger patients because the annulus is strong and intact, and thus nuclear material herniates through the weaker endplate. They are associated with lumbar degenerative change, but are not themselves an independent risk factor for back pain[24].

Ligamentum flavum hypertrophy (LFH) was noted in $9 \%$. It was mostly seen at lower spinal levels (predominantly at L4/L5 and L5/S1), similar to study conducted by Saleem et al [17]. In the study done by PY Yong et al [16]14.0\% manifested LFH.Vertebral compression was seen in 5.7\% of the study population with anterior wedge collapse accounting for majority (90\%) of cases. Mustapha et al [8] found wedge collapse in $1.97 \%$. Higher value in the present study could be due to more number of female patients (predisposed to osteoporosis), whereas male predominance was present in the study of Mustapha et al [8]. Lumbosacral transitional vertebrae in the form of sacralization of L5 vertebra was noted in $4 \%$. No cases of lumbarization of S1 were seen in present study. Study conducted by S Verma [25] on MRI findings in $20-40$ year old patients with low back pain revealed sacralization of L5 in $9 \%$ of patients. The prevalence of LSTV reported in the literature ranges from 4 to over $35 \%$ [26].

Spondylolisthesis was noted in $3 \%$ of cases in this study, with female predominance $(2.5 \%$ female, $0.5 \%$ male). Half of the listhesis cases involved L4-L5 level and half L5-S1 level. It is similar to study of Rai et al [14] who reported $2 \%$ cases of spondylolisthesis, all involving L4/L5 level. Female predominance was noted (83\%).Amongst females, most frequent level of listhesis was L5 over S1 (60\%).This is similar to study of Dootchai et al [27]. Higher prevalence of listhesis is most likely due to hormonal effects on soft tissue laxity [28].

\section{Conclusion}

Role of diagnostic imaging in patients with low back pain is to provide accurate anatomic information which in turn affects the management. This study highlights the various patterns of degenerative spinal disease associated with low back pain. Lumbar disc degeneration (LDD) is the most common pattern which consists of disc dessication (66\%) disc bulges (85\%) and disc herniations $(25 \%)$. LDD is most common at the L4/L5 followed by L5/S1 level. Posterocentral disc protrusion is the commonest type of disc herniation. Other findings associated with degenerative spine/low back ache were osteophytes, flaval hypertrophy, vertebral compression, Schmorl's nodes, spondylolisthesis ,facetal joint hypertrophy and spinal stenosis.

\section{References}

[1] Woolf AD, Pfleger B. Burden of major musculoskeletal conditions. Bulletin of the World Health Organization,81 (9), 2003, 646656.

[2] Robert W. Teasell, Kevin White. Clinical approaches to low back pain Part 1. Epidemiology, diagnosis, and prevention. Canadian Family Physician, 40, 1994.481-86.

[3] D. L. Kent, D. R. Haynor, E. B. Larson, R. A. Deyo. Diagnosis of lumbar spinal stenosis in adults: a meta analysis of the accuracy of CT, MR, and myelography. Am J Roentgenol. 158(5),1992, 1135-1144.

[4] M. Nazeer, S.M Rao, S. Soni, M Ravinder, T Ramakranthi, S. Bhupathi. Low Back Pain in South Indians: Causative Factors and Preventive Measures. Sch. J. App. Med. Sci, 3(1D) 2015, 234-243.

[5] Wijnhoven HA, de Vet HC, Picavet HS. Prevalence of musculoskeletal disorders is systematically higher in women than in men. Clin J Pain, 22, 2006:717-24.

[6] Prasad R, Hoda M, Dhakal M, Singh K, Srivastava A, Sharma V. Epidemiological characteristics of lumbar disc prolapse in a tertiary care hospital. Internet J Neurosurg 3(1).2005.

[7] Younis F, Shahzad R, Rasool F. Correlation of magnetic resonance patterns of lumbar disc disease with clinical symptomatology of patients. Annals of King Edward Medical University 17(1),2011.41-47

[8] Mustapha Z, Ahmadu MS, Abbas Ali A, Ibrahim K \& Okedayo M. Patterns of Requests and Findings in Magnetic Resonance Imaging (MRI) of the Lumbosacral Spine at University of Maiduguri Teaching Hospital, Northeastern Nigeria. IOSR Journal of Dental and Medical Sciences 11(4),2013,18-24.

[9] Iftikhar AB, Noman M, Javed M, Faisal N, Humayun P. Magnetic Resonance patterns of lumbar disc disease. J Rawal Med Coll, 8, 2004, 53-6.

[10] Lipson SJ, Muir H. Experimental intervertebral disc degeneration: Morphologic and proteoglycan changes over time. Arthritis Rheum ,24,1981,12-21. 
[11] Osama Al-saeed, Khaled Al-Jarallah, Maryam Raees, Mehraj Sheikh, Mohammed Ismail, Reji Athyal. Magnetic Resonance Imaging of the Lumbar Spine in Young Arabs with Low Back Pain. Asian Spine Journal 6 (4), 2012 $249 \sim 256$.

[12] M. Jacob, L.O. Akoko, R.R. Kazema. Lumbar disc degenerative disease: magnetic resonance imaging findings in patients with low back pain in Dar Es Salaam. East \& Central African Journal of Surgery, 20(1), 2015 .122-131.

[13] Dr. Felix U. Uduma, Dr. Pierre Ongolo, Dr. George Assam, Dr. Pius Fokam, Dr. Mathieu Motah. Evaluation of Pattern of Magnetic Resonance Images of Lumbo-Sacral Spine in Cameroon - A Pioneer Study. Global Journal of Medical research ,11 (1). 2011.2

[14] Garjesh Singh Rai, Alankrita Mehra, Tribhuwan Narayan Singh Gaur. A prospective study of magnetic resonance imaging findings in patients of chronic low back pain: a clinico-radiological correlation. Int J Res Med Sci. ,4(1):2016,47-56.

[15] Van der Kraan PM, van den Berg WB. Osteophytes: relevance and biology. Osteoarthritis cartilage. ,15(3), $2007,237-44$.

[16] PY Yong, NAA Alias, IL Shuaib. Correlation of Clinical Presentation, Radiography, and Magnetic Resonance Imaging for Low Back Pain — a Preliminary Survey. J HK Coll Radiol 2003;6:144-151.

[17] Saleem S, Aslam HM, Rehmani MAK, Raees A, Alvi AA, Ashraf J. Lumbar Disc Degenerative Disease: Disc Degeneration Symptoms and Magnetic Resonance Image Findings. Asian Spine Journal. 27(4),2013, 322-334.

[18] Schellhas KP, Pollei SR, Gundry CR, Heithoff KB. Lumbar disc high-intensity zone, correlation of magnetic resonance imaging and discography. Spine, 21, 1996:79-86.

[19] Lam KS, Carlin D, Mulholland RC. Lumbar disc high-intensity zone: the value and significance of provocative discography in the determination of the discogenic pain source. Eur Spine J ,9 2000, 36-41.

[20] Peng B, Hou S, Wu W, Zhang C, Yang Y. The pathogenesis and clinical significance of a high-intensity zone (HIZ) of lumbar intervertebral disc on MR imaging in the patient with discogenic low back pain. Eur Spine J ,15 2006, 583-7.

[21] Ricketson R, Simmons JW, Hauser BO. The prolapsed intervertebral disc. The high-intensity zone with discography correlation. Spine 1,21(23) 1996, 2758-62.

[22] Smith BM, Hurwitz EL, Solsberg D, Rubinstein D, Corenman DS, Dwyer AP, et al. Interobserver reliability of detecting lumbar intervertebral disc high-intensity zone on magnetic resonance imaging and association of high-intensity zone with pain and annular disruption. Spine, 23 1998,2074-2080.

[23] Rankine JJ, Gill KP, Hutchinson CE, Ross ER, Williamson JB. The clinical significance of the high-intensity zone on lumbar spine magnetic resonance imaging. Spine 24, 1999, 1913-1919.

[24] F. M. K. Williams, N. J. Manek, P. N. Sambrook, T. D. Spector, A. J. Macgregor. Schmorl's nodes: common, highly heritable, and related to lumbar disc disease. Arthritis Rheum. 15, 57(5), 2007, 855-860.

[25] Verma SR, Gupta PK, Munshi A, Goyal P, Verma SC. A retrospective analysis of magnetic resonance imaging findings in $20-40$ year old patients with low back pain: experience at a semi-urban tertiary healthcare centre in Northern India. The Internet Journal of Spine Surgery, 6(4), 2011,1937-827045.

[26] Johannes L. BRON, Barend J. Van Royen, Paul I. J. M. Wuisman. The clinical significance of lumbosacral transitional anomalies. Acta Orthop. Belg., 73,2007, 687-695.

[27] Dootchai Chaiwanichsiri, Araya Jiamworakul , Sutthichai Jitapunkul. Lumbar Disc Degeneration in Thai Elderly: A PopulationBased Study. J Med Assoc Thai Vol. 90 No. 112007.

[28] Vogt MT, Rubin DA, Palermo L, Christianson L, Kang JD, Nevitt MC, et al. Lumbar spine listhesis in older African American women. Spine J , 3,2003, 255-61.

Table 1: Frequency of various abnormalities in degenerative spine disease

\begin{tabular}{|l|l|}
\hline Type of abnormality & Frequency \\
\hline Disc Bulge & $85 \%$ \\
\hline Disc dessication & $66 \%$ \\
\hline Disc Protrusion & $23 \%$ \\
\hline Disc extrusion & $2 \%$ \\
\hline HIZ/Annular tear & $22 \%$ \\
\hline Schmorl's nodes & $10 \%$ \\
\hline Osteophytes & $52 \%$ \\
\hline Facetal arthropathy & $27 \%$ \\
\hline Modic changes & $21 \%$ \\
\hline Flaval hypertrophy & $9 \%$ \\
\hline Spinal stenosis & $50.5 \%$ \\
\hline Vertebral compression & $5.7 \%$ \\
\hline Transitional vertebra & $4 \%$ \\
\hline Spinal listhesis & $3 \%$ \\
\hline
\end{tabular}

Table 2: Age and levels of disc bulge in degenerative spine

\begin{tabular}{|l|l|l|l|l|l|}
\hline AGE & L1-L2 & L2-L3 & L3-L4 & L4-L5 & L5-S1 \\
\hline $21 Y-30 Y$ & 0 & 0 & 18 & 26 & 21 \\
\hline $31 Y-40 Y$ & 3 & 11 & 26 & 37 & 28 \\
\hline $41 Y-50 Y$ & 4 & 9 & 14 & 30 & 19 \\
\hline $51 Y-60 Y$ & 2 & 4 & 16 & 22 & 14 \\
\hline $61 Y-70 Y$ & 4 & 8 & 19 & 21 & 12 \\
\hline $71 Y-80 Y$ & 0 & 1 & 2 & 2 & 2 \\
\hline Total & 13 & 33 & 95 & 138 & 96 \\
\hline
\end{tabular}




\begin{tabular}{|c|c|c|c|c|c|c|}
\hline Age in years & central & $\begin{array}{l}\text { Right } \\
\text { paracentral }\end{array}$ & $\begin{array}{l}\text { Left } \\
\text { paracentra } \\
1 \\
\end{array}$ & $\begin{array}{l}\text { Central \& Right } \\
\text { para central }\end{array}$ & $\begin{array}{l}\text { Central \& } \\
\text { left } \\
\text { paracentral }\end{array}$ & $\begin{array}{l}\text { Grand } \\
\text { Total }\end{array}$ \\
\hline 21 to 30 & 5 & 1 & 2 & 0 & 0 & $8(18 \%)$ \\
\hline 31 to 40 & 8 & 1 & 2 & 0 & 2 & $13(30 \%)$ \\
\hline 41 to 50 & 4 & 1 & 2 & 0 & 2 & $9(20.5 \%)$ \\
\hline 51 to 60 & 4 & 0 & 0 & 1 & 0 & $5(11 \%)$ \\
\hline 61 to 70 & 7 & 0 & 0 & 2 & 0 & $9(20.5 \%)$ \\
\hline 71 to 80 & 0 & 0 & 0 & 0 & 0 & 0 \\
\hline Grand Total & 28 & 3 & 6 & 3 & 4 & 44 \\
\hline
\end{tabular}

Table 4: Different Patterns of spinal stenosis

\begin{tabular}{|l|l|l|l|}
\hline Pattern & Male & Female & Total(Vertebral Levels In 93 Patients) \\
\hline Central & 8 & 6 & $14(11 \%)$ \\
\hline B/L Lateral Recess & 36 & 42 & $78(63.4 \%)$ \\
\hline Right Lateral Recess & 0 & 1 & 1 \\
\hline Left Lateral Recess & 0 & 0 & 0 \\
\hline B/L Foraminal & 14 & 11 & $25(20 \%)$ \\
\hline Right Foraminal & 1 & 0 & 1 \\
\hline Left Foraminal & 3 & 1 & 4 \\
\hline
\end{tabular}

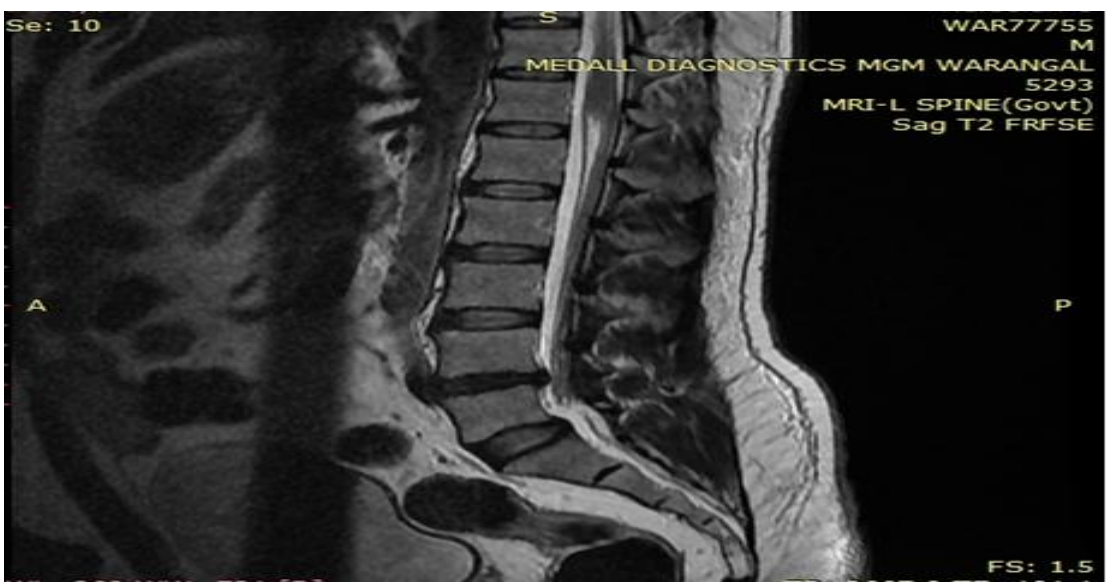

Figure 1: A sagittal T2W image showing disc dessication and bulge at L4-L5 level with spinal canal stenosis

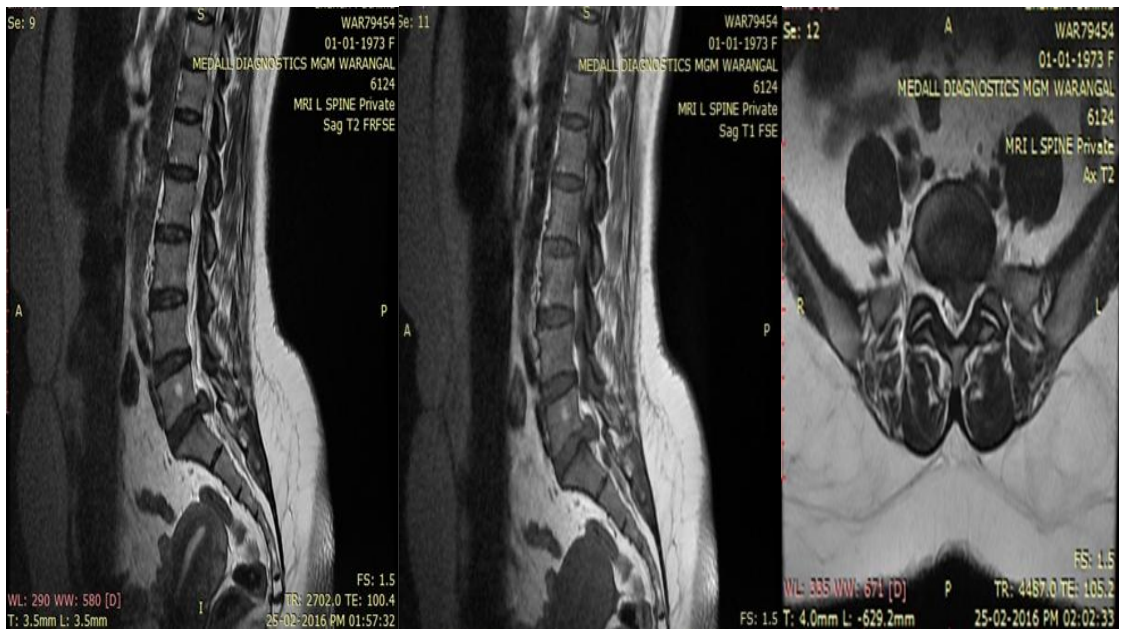

Figure 2: Sagittal T2W, T1W, Axial T2W images showing an extrusion of L5-S1 disc causing significant compression on cauda equina and left lateral recess. Hemangioma in L5 vertebral body. 


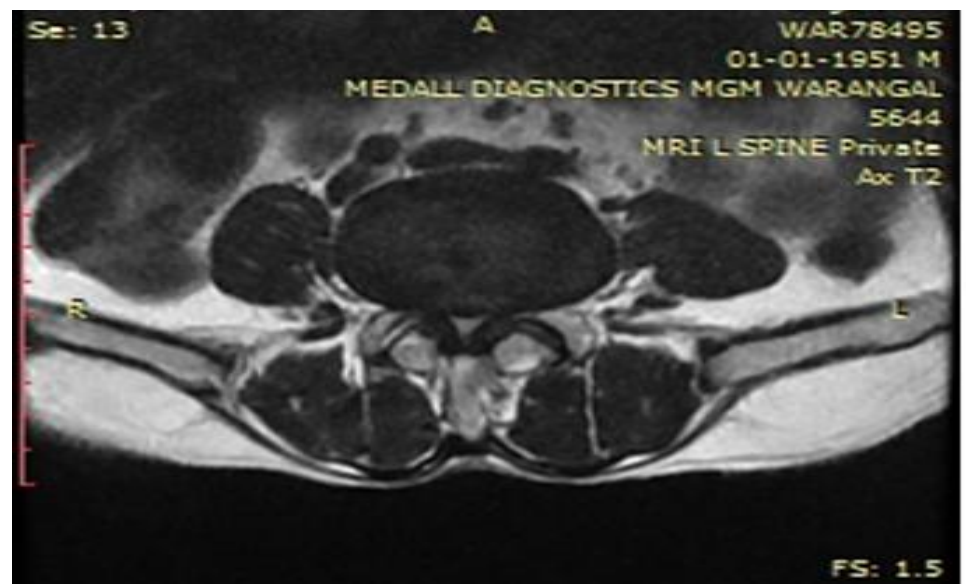

Figure 3: Axial T2W image showing a diffuse disc bulge, flaval thickening, and facetal arthropathy.

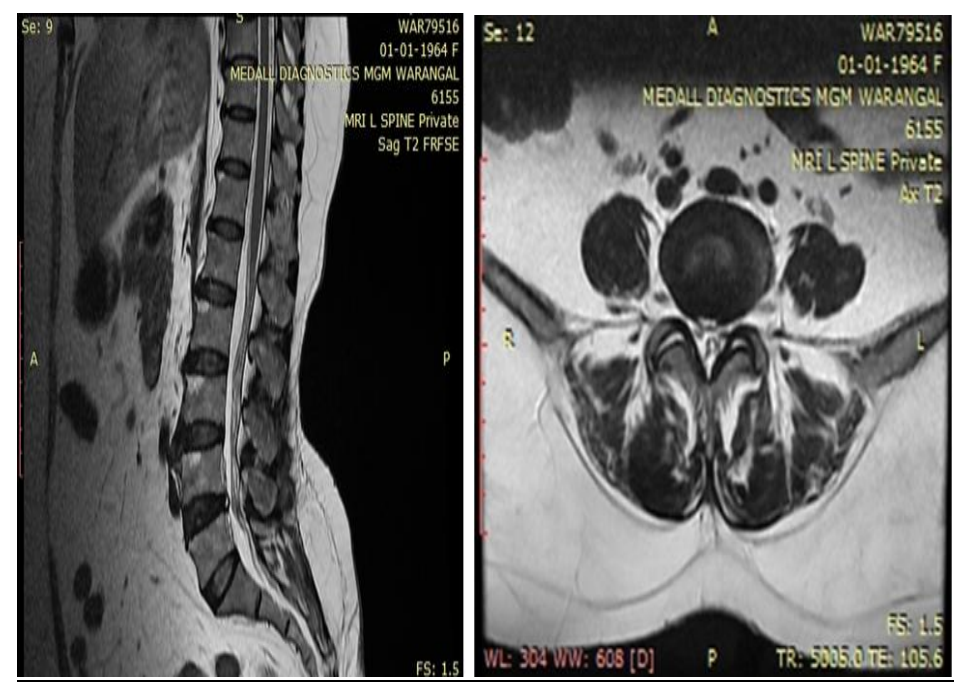

Figure 4: Sagittal and axial T2W images showing HIZ (high intensity zone-annular tear)at L4-L5 disc level posteriorly and type II modic end plate changes at L2, L3, and L4 superior vertebral end plates. 